\title{
Modality-specific Alpha Modulations Facilitate Long-term Memory Encoding in the Presence of Distracters
}

\author{
Haiteng Jiang, Marcel A. J. van Gerven, and Ole Jensen
}

\begin{abstract}
It has been proposed that long-term memory encoding is not only dependent on engaging task-relevant regions but also on disengaging task-irrelevant regions. In particular, oscillatory alpha activity has been shown to be involved in shaping the functional architecture of the working brain because it reflects the functional disengagement of specific regions in attention and memory tasks. We here ask if such allocation of resources by alpha oscillations generalizes to long-term memory encoding in a cross-modal setting in which we acquired the ongoing brain activity using magnetoencephalography. Participants were asked to encode pictures while ignoring simultaneously
\end{abstract}

\section{INTRODUCTION}

In our daily life, our sensory systems are bombarded by sensory information of which only a small fraction needs to be remembered, for example, when reading a paper while ignoring colleagues conversing in the background. This implies that we rely on mechanisms that maintain relevant information to be encoded and, just as importantly, mechanisms for suppressing irrelevant information to be ignored.

Oscillatory activity in the alpha band $(8-12 \mathrm{~Hz})$ has been proposed to play an important role in the engagement and disengagement of sensory areas depending on task demands (Klimesch, 2012; Foxe \& Snyder, 2011; Klimesch, Sauseng, \& Hanslmayr, 2007). The alpha rhythm reflects active inhibition or top-down processing and might serve to direct information flow through the brain and allocate resources to relevant regions (Jensen \& Mazaheri, 2010). Various studies on attention and working memory support this mechanism. For example, hemispherically lateralized alpha activity is observed in visual attention tasks, where alpha power decreases contralateral to the attended side while it relatively increases contralateral to the ignored side (Kelly, Lalor, Reilly, \& Foxe, 2006; Worden, Foxe, Wang, \& Simpson, 2000). This lateralized alpha activity correlates with visual detection performance (Handel, Haarmeier, \& Jensen, 2011; Thut, Nietzel, Brandt, \& Pascual-Leone, 2006). A similar phenomenon is found

Radboud University Nijmegen presented words and vice versa. We quantified the brain activity during rehearsal reflecting subsequent memory in the different attention conditions. The key finding was that successful long-term memory encoding is reflected by alpha power decreases in the sensory region of the to-be-attended modality and increases in the sensory region of the to-be-ignored modality to suppress distraction during rehearsal period. Our results corroborate related findings from attention studies by demonstrating that alpha activity is also important for the allocation of resources during long-term memory encoding in the presence of distracters. during saccade planning (Medendorp et al., 2007) and somatosensory working memory tasks (Haegens, Luther, \& Jensen, 2012; Haegens, Handel, \& Jensen, 2011). In cross-modal paradigms, visual alpha appears when attention is allocated to the auditory modality (Fu et al., 2001; Foxe, Simpson, \& Ahlfors, 1998; Adrian, 1944). Furthermore, increased alpha activity in supramarginal gyrus (SMG) has been reported when attention is deployed to the visual modality (Mazaheri et al., 2014). Since a number of neuroimaging studies have indicated a critical functional role of the SMG in higher-level auditory processing (Sabri et al., 2008; Gaab, Gaser, Zaehle, Jancke, \& Schlaug, 2003; Celsis et al., 1999), it was suggested that the increase of alpha power in the SMG reflects inhibition of nonvisual processing. Moreover, increased alpha power after a cue to ignore the item can aid STM, supposedly filtering out incoming information (Dube, Payne, Sekuler, \& Rotello, 2013; Payne, Guillory, \& Sekuler, 2013; Sauseng et al., 2005). Likewise, a recent long-term memory (LTM) study demonstrated that visual alpha activity could serve to block memory encoding when directed by a cue (Park et al., 2014). However, little is known about the role of alpha activity during LTM encoding in the presence of distracters in a cross-modal setting.

We hypothesize that alpha oscillations play a functional role for the allocation of resources in a cross-modal memory task. In our paradigm, we presented participants with auditory (words) and visual (pictures) stimuli simultaneously. Participants were instructed in blocks to attend to one of the modalities while ignoring the other. 
Consequently, we expect modulations of alpha activity during encoding in extended visual and auditory areas to be predictive of subsequent LTM performance. To investigate this, we recorded ongoing brain activity using magnetoencephalography (MEG). This allows for characterizing the temporal characteristics of oscillatory brain activity in the involved brain regions.

\section{METHODS}

\section{Participants}

Twenty participants (21-28 years, 12 women) gave written informed consent to participate in this study. All participants were reported to be right-handed, native Dutch speakers, and had no history of neurological or psychiatric disorders. The study was approved by the local ethics committee (CMO Region Arnhem/Nijmegen).

\section{Stimuli}

We selected 648 high-frequency ( $>90$ occurrences per million) concrete Dutch nouns with a word length of 2-13 letters from the Celex database (celex.mpi.nl). High-quality auditory stimuli were recorded by a native female Dutch speaker and further trimmed to $600 \mathrm{msec}$ duration using the Goldwave software (www.goldwave.com). For the visual stimuli, images that matched the words were selected from the Internet. These images were resized to $400 \times$ 300 pixels with Adobe Photoshop CS2, converted to grayscale, and normalized with the SHINE toolbox to have matching luminance (Willenbockel et al., 2010).

The words were presented via MEG-compatible wire tubes, and visual images were projected onto the screen using a liquid crystal display video projector (Sanyo Pro Xtrax Multiverse, Secaucus, NJ; refresh rate of $60 \mathrm{~Hz}$ ). The visual angle of the stimuli was $8^{\circ}$ horizontally. The volume was adjusted individually to make sure that participants could hear the auditory stimuli clearly and feel comfortable at the same time. The experiment was controlled by Psychtoolbox software (psychtoolbox.org).

\section{Experimental Design}

The experiment consisted of an encoding and a recognition session (Figure 1). The encoding session had six blocks, alternating between Attend-Visual and AttendAuditory. Each block had 36 trials in which visual and auditory stimuli were presented simultaneously (pictures and spoken words were always incongruent). A trial started with the presentation of a fixation cross $(400 \mathrm{msec})$. Subsequently, the stimuli were presented $(600 \mathrm{msec})$, followed by a 3000-msec rehearsal period. In the Attend-Visual condition, participants had to encode the pictures while not paying attention to the words (Unattended-Auditory). In contrast, in the Attend-Auditory condition, the words had to be encoded while ignoring the images (Unattended-

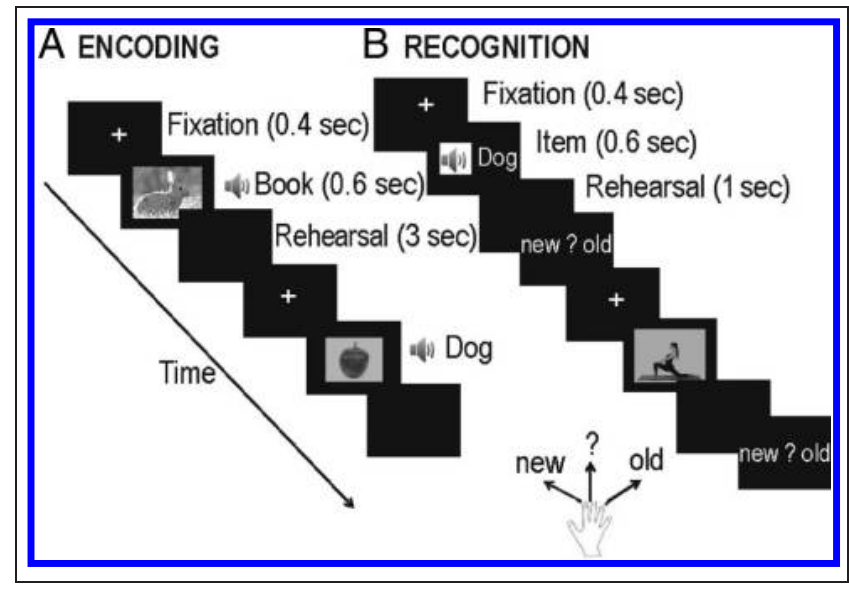

Figure 1. A cross-modal LTM paradigm. (A) In the encoding session, participants were presented with auditory (words) and visual (pictures) stimuli simultaneously and cued in blocks to attend one of the modalities. In the Attend-Visual condition, participants had to encode visual images while ignoring the concurrently presented words, and vice versa in the Attend-Auditory condition. Before each block of 36 stimuli, a visual cue indicated which modality to attend. In total, 216 pictures and 216 words were presented of which half were attended. (B) In the recognition session, previously presented items were randomly intermixed with 108 new visual and 108 new auditory stimuli (foils). Participants were instructed to indicate if they had seen a given item in the encoding session by pressing one of three buttons corresponding to "new," "don't know," or "old."

Visual). Before each block, a visual cue indicated whether to encode pictures or words. The first block (AttendAuditory or Attend-Visual) was counterbalanced across participants. The encoding session lasted about $20 \mathrm{~min}$ in which respectively 216 words and pictures were supposed to be encoded.

The recognition task session followed after a 10 -min break in the MEG system. During this session, 216 images and 216 words presented during the encoding session were randomly intermixed with 108 new images and 108 new words (foils). Behavioral responses were recorded using right-hand button presses. Participants were instructed to give an "old" response by pressing a right button for stimuli they were confident to have seen in the encoding session. For stimuli they considered "new," they had to press the left button. Participants were asked to push the middle button when they were uncertain about stimulus novelty. Before the full experiment, the participants performed a practice session to become familiar with the task. Thus, participants were aware that they later would be tested on the cued modality during the encoding session. This promoted that they allocated attention according to the cue instructions.

\section{Data Acquisition}

MEG data were recorded with a 275-sensor CTF Omega whole-head gradiometer system (VSM MedTech, Coquitlam, $\mathrm{BC}$, Canada). Two sensors were not functioning because 
of technical problems and were ignored in the analysis. The data were sampled at $1200 \mathrm{~Hz}$ and low-pass filtered at $300 \mathrm{~Hz}$. Head localization was done before and after the experiment using marker coils that were placed at the cardinal points of the head (nasion, left and right ear canal). The head position was continuously monitored online and adjusted if head motion exceeded $5 \mathrm{~mm}$ (Stolk, Todorovic, Schoffelen, \& Oostenveld, 2013). The magnetic fields produced by these coils were used to estimate the position of the participant's head with respect to the MEG sensor array. In addition, bipolar electrodes were attached to record the electrocardiogram and EOG.

After the MEG recording, participants underwent an anatomical MRI scan using a 1.5-T Siemens Magnetom Avanto MRI scanner (Siemens Healthcare, Erlangen, Germany). To realign the MEG source reconstructions and the structural MRI data, earplugs containing oil with vitamin E were placed in the ear canals during MRI acquisition.

\section{Data Analysis}

The data acquired in the encoding session were analyzed using the FieldTrip toolbox (Oostenveld, Fries, Maris, \& Schoffelen, 2011). First, trials with high variance because of MEG sensor jumps, movement, or muscle artifacts were removed based on visual inspection (on average $6 \%$ of the trials). Furthermore, we used independent component analysis to identify components reflecting eye artifacts. These components were then removed from the data. Following artifact rejection, the trials were sorted according to the conditions. Three participants were excluded because of abundant eye blinking, muscle activity, and movement artifacts during the recording sessions. One more participant was excluded because of electronic failure, leaving data sets for 16 participants (nine women).

\section{Spectral Analysis}

Epochs of $5 \mathrm{sec}$, including a 400-msec fixation period preceding the onset of stimulus presentation, $600 \mathrm{msec}$ of stimuli presentation, and $3000 \mathrm{msec}$ of rehearsal period were used in the time-frequency (TF) of power analysis. Additionally, 500-msec time windows preceding the baseline and following the end of the rehearsal period were included to avoid edge effects in the subsequent TF analysis. A time-frequency representation (TFR) was calculated for each trial using a fast Fourier transform using a taper approach applied to a sliding time window moving in 50-msec increments. For the analysis in the $5-30 \mathrm{~Hz}$ range, we applied an adaptive time window of five cycles for each frequency (e.g., $\Delta T=500 \mathrm{msec}$ for $f=10 \mathrm{~Hz}$ ). Before the Fourier transform, a Hanning taper of the same length was multiplied to each segment. The power was averaged over trials for each condition first and then a logarithmic (base 10) transform was applied to reduce interparticipant variance. The difference between conditions was calculated as log-transformed power difference. Because we applied a block design, there might have been systematic differences in the baseline intervals. Thus, the TFRs were not baseline-corrected.

\section{Source Analysis}

To identify the sources of the oscillatory activity, we applied a beamforming approach in the frequency domain based on an adaptive spatial filter approach (Gross et al., 2001). Cross-spectral density matrices were calculated from the Fourier transformed data for each condition. Realistically shaped single-shell descriptions of participants' brains were constructed from each individual's anatomical MRI. The brain volume of each individual participant was divided into a grid with a $0.8-\mathrm{cm}$ resolution and normalized to the template MNI brain (International Consortium for Brain Mapping, Montreal Neurological Institute, Canada) using SPM8 software (www.fil.ion.ucl.ac.uk/spm). The lead fields were calculated for each grid point. Then, a spatial filter was constructed for each grid point using the cross-spectral density matrices for the frequency of interest and the lead fields. The spatial distribution of power of oscillatory activity was estimated in each condition, whereas the cross-spectral densities were calculated for the Fourier transformed combined data.

The grid points ("voxels") included in ROIs were selected on the basis of Anatomy toolbox in SPM8 (Tzourio-Mazoyer et al., 2002). We used the following two ROIs: occipital cortex (including bilateral occipital superior cortex, bilateral occipital middle cortex, and bilateral occipital inferior cortex) and SMG (bilateral SMG) for later cluster analysis.

\section{Statistical Analysis}

Nonparametric cluster-based randomization tests were applied to the sensor and source data (Maris \& Oostenveld, 2007). This test controls for Type I errors in situations involving multiple comparisons by clustering neighboring sensors or gridpoints. We averaged over the time and frequency of interest. Sensors/gridpoints become part of a cluster when the dependent samples $t$ value between the conditions exceeded an a priori threshold $(p<.05)$, and these were subsequently clustered on the basis of spatial adjacency. The cluster-level statistic was defined as the sum of the $t$ values over the sensors/grid points in the cluster. The cluster with the maximum sum was used as a test statistic. By randomizing the data across the two conditions and recalculating the test statistic 1000 times, we obtained a reference distribution of maximum cluster $t$ values to evaluate the statistic of the actual data.

\section{RESULTS}

\section{Behavioral Outcomes}

Behavioral results from the recognition session are reported in Figure 2. Trials in the recognition session were 


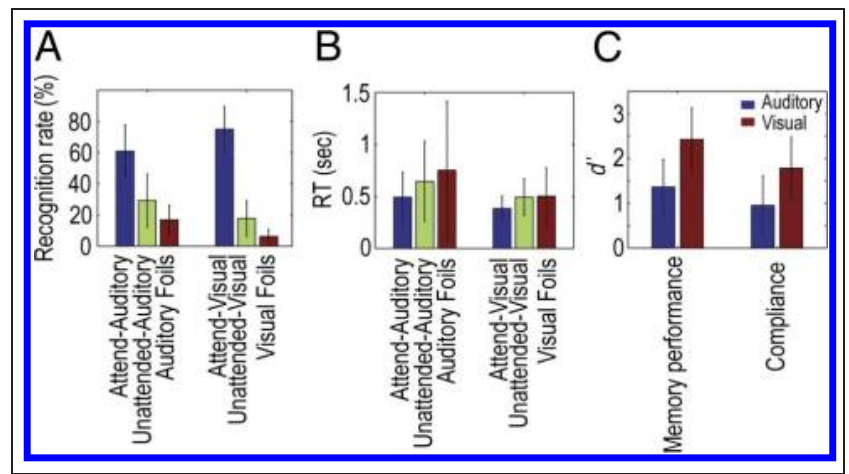

Figure 2. Behavioral results from the recognition session. (A) Recognition rates for the different conditions. The HIT rate was significantly higher for attended items compared with unattended items in both the visual and auditory conditions. The FA rate for the foils was relatively low. (B) RT for the different conditions. Participants respond faster in visual conditions than auditory conditions. (C) The left bars reflect the memory performance ( $d^{\prime}$ calculated for Attend-HIT vs. FA). The right bars reflect compliance defined as the $d^{\prime}$ calculated for Attend-HIT versus Unattended-HIT (i.e., Unattended-HIT trials are considered FA in the $d^{\prime}$ measure)

classified as correctly identified stimuli (HIT), unrecognized old stimuli (MISS), correctly rejected new stimuli (CR), and new stimuli incorrectly identified as "old," that is, false alarm (FA). A $2 \times 3$ repeated-measures ANOVA analysis of recognition rate with the factors Modality (auditory, visual) and Attention (attend, unattended, foils) revealed significant main effects for Attention, $F(2,30)=$ $186.82, p<.001$, and significant Modality $\times$ Attention interaction, $F(2,30)=11.25, p=.001$ (Greenhouse-Geisser Corrected). There was no main effect for Modality. The recognition rate for the attended stimuli (auditory: $60.8 \pm$ $17.0 \%$; visual: $75 \pm 14.7 \%$ ) was significantly greater than for the unattended stimuli as shown in Figure 2A. These results demonstrate that participants remembered more attended compared with unattended items. Furthermore, the low FA rate for the foils indicated that random guessing was relatively low during retrieval. Regarding the RT (Figure 2B), there was a main effect for Modality, $F(1,15)=10.40, p=.021$, but only a trend for Attention, $F(2,30)=4.47, p=.061$. No significant Modality $\times$ Attention interactions were found, $F(2,30)=0.64, n s$. Participant response was faster in visual conditions than auditory conditions (Attend-Visual: $0.38 \pm 0.12 \mathrm{sec}$; Attend-Auditory: $0.49 \pm 0.24 \mathrm{sec}$; Unattended-Visual: $0.49 \pm 0.18 \mathrm{sec}$; Unattended-Auditory: $0.64 \pm 0.38 \mathrm{sec}$; Foils-Visual: $0.50 \pm$ $0.28 \mathrm{sec}$; Foils-Auditory: $0.75 \pm 0.66 \mathrm{sec}$ ). Memory performance was further assessed by the standard $d^{\prime}$ measure calculated for Attend-HIT versus FA rates, and "compliance" was computed by the $d^{\prime}$ measure between Attend-HIT and Unattended-HIT rates in visual and auditory modalities, respectively (see Park et al., 2014, for the compliance measure). The higher $d^{\prime}$ value (Figure 2C) for the visual modality suggests that the visual task was easier than the auditory task. Moreover, the high $d^{\prime}$ values for compliance indicated that participants did follow the attention cueing.

\section{Temporal Modulations in Alpha Power Suggest a Two-stage Process during LTM Encoding}

We first set out to investigate the difference in oscillatory activity when comparing Attend-Visual with Attend-Auditory memory encoding. The TFRs of power were calculated per trial and averaged for the different conditions. Figure 3A shows two distinct phases of modulations in the alpha band during rehearsal. During the first second after stimulus offset, alpha power was higher in the visual as compared with the auditory condition. Subsequently, it was higher in the auditory as compared with the visual condition. To

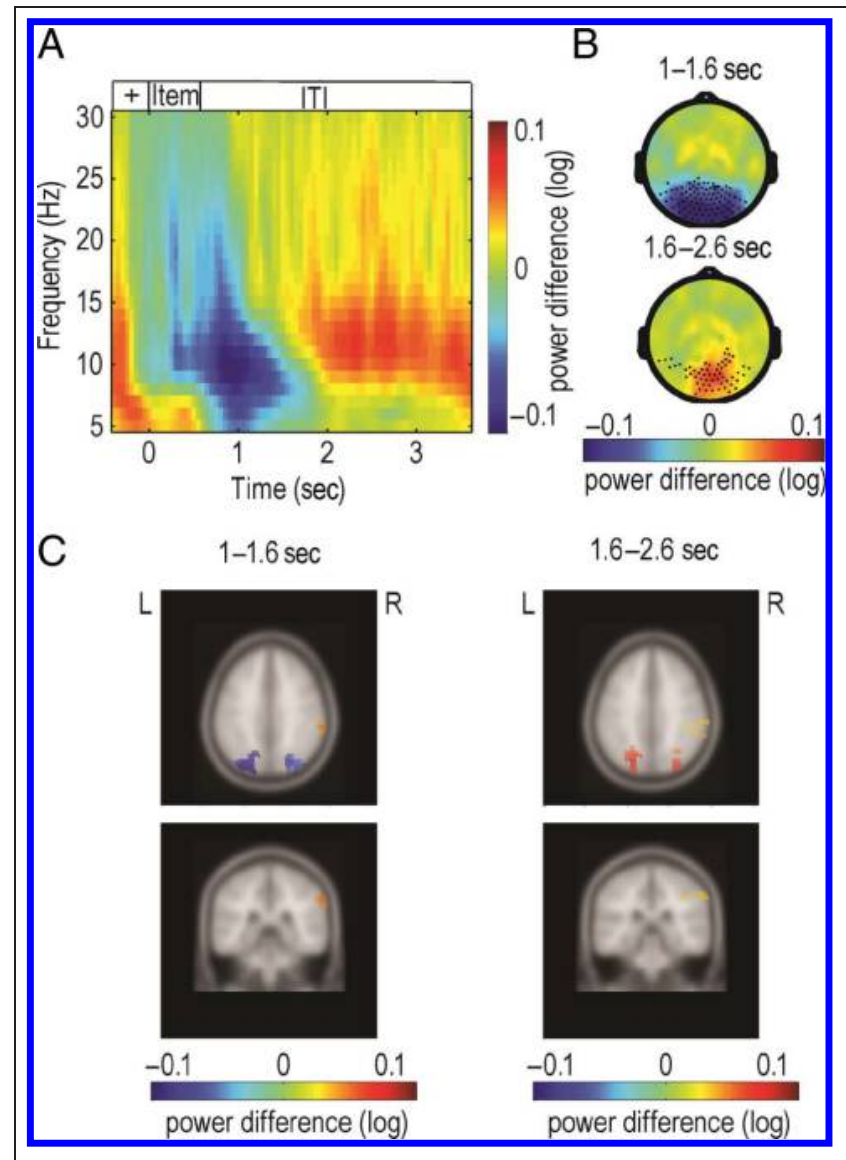

Figure 3. Alpha band activity for the Attend-Visual condition compared with the Attend-Auditory condition. (A) Relative differences in TFRs of power for the contrast Attend-Visual versus Attend-Auditory obtained for posterior sensors. In the subsequent analyses in this article, we considered the alpha activity in the 1-1.6 sec and 1.6-2.6 sec time window. (B) Scalp topographies of the difference in $8-12 \mathrm{~Hz}$ power $1-1.6 \mathrm{sec}$ and $1.6-2.6 \mathrm{sec}$ after stimulus onset. Sensors marked with dots are part of the significant clusters obtained in a permutation analysis controlling for multiple comparisons $(p<.05)$. (C) Source reconstruction of the difference in alpha power modulation in visual cortex and SMG (Attend-Visual vs. Attend-Auditory). The regions of significant difference were identified using a cluster-level permutation test $(p<.05)$. This allowed us to control for multiple comparisons in visual cortex and bilateral SMG, respectively. Left (1-1.6 sec early rehearsal period): negative significant clusters in visual cortex and positive significant clusters in right SMG. Right (1.6-2.6 sec late rehearsal period): positive significant clusters in visual cortex and right SMG. 
avoid interference from early visual evoked responses and to dissociate early and late effects, we focused the subsequent analysis on the following two time windows: early rehearsal (1-1.6 sec after stimulus onset) and late rehearsal (1.6-2.6 sec after stimulus onset). A nonparametric permutation analysis resulted in a cluster of sensors located over occipital-parieto regions in both intervals (1-1.6 sec: $p=.003$; $1.6-2.6$ sec: $p=.048$; Figure $3 \mathrm{~B})$. Source reconstruction using a beamforming approach revealed that the early power decrease was produced in visual areas and an increase in right SMG (visual areas: $p=.003$; SMG: $p=$ .046 ; Figure $3 \mathrm{C}$, left). This suggests that alpha power decreases in the visual system to support memory encoding of visual compared with auditory items. We speculate that the right SMG alpha power increases to prevent auditory encoding. In the late rehearsal period, alpha power showed a relative increase in visual cortices and SMG (visual areas: $p=.043$; SMG: $p=.048$; Figure 3C, right). This finding suggests that the visual cortex and right SMG is blocked by an alpha increase during the later part of the rehearsal period when comparing visual to auditory items. This might reflect the disengagement of occipital areas preventing interference from the visual system. In short, this modulation of alpha power suggests two phases during LTM encoding. Note that this finding just as well also reflects an early increase in occipital alpha associated with the encoding of auditory items. We will next consider the Attend-Visual and Attend-Auditory conditions in more detail to bring further insight into this issue.

\section{Subsequent Memory Effect for Visually Attended Items}

Next, we set out to identify the temporal dynamics reflecting subsequent memory effect (SME) for visually attended items. We chose the time windows based on the findings from Figure 3. Note that this contrast (auditory versus visual) is orthogonal to the subsequent memory contrast. Figure 4A shows the TFR of power for later remembered (HIT) versus later forgotten items (MISS) for the visually attended items (log power difference). We refer to this contrast as the Attend-Visual SME. The TFRs revealed an initial decrease in alpha activity for the Attend-Visual SME over posterior sensors during early rehearsal (Figure 4A). A cluster-based randomization test showed that the effect was significant in the alpha band activity in occipital sensors in the early rehearsal period ( $p=.008$; Figure $4 \mathrm{~B}$ ). Sources reflecting the relative alpha power decrease for the Attend-Visual SME were located in the visual cortex $(p=.002$; Figure $4 \mathrm{C}$, left). We did not identify a subsequent effect in the later interval. These findings suggest that successful memory encoding relies on engagement of occipital areas as reflected by an alpha band decrease. Besides, alpha sources reflecting an increase were found in the right SMG $(p=.019$; Figure $4 \mathrm{C}$, right), indicating disengagement of the auditory system. The latter effect was, however, not reflected at the

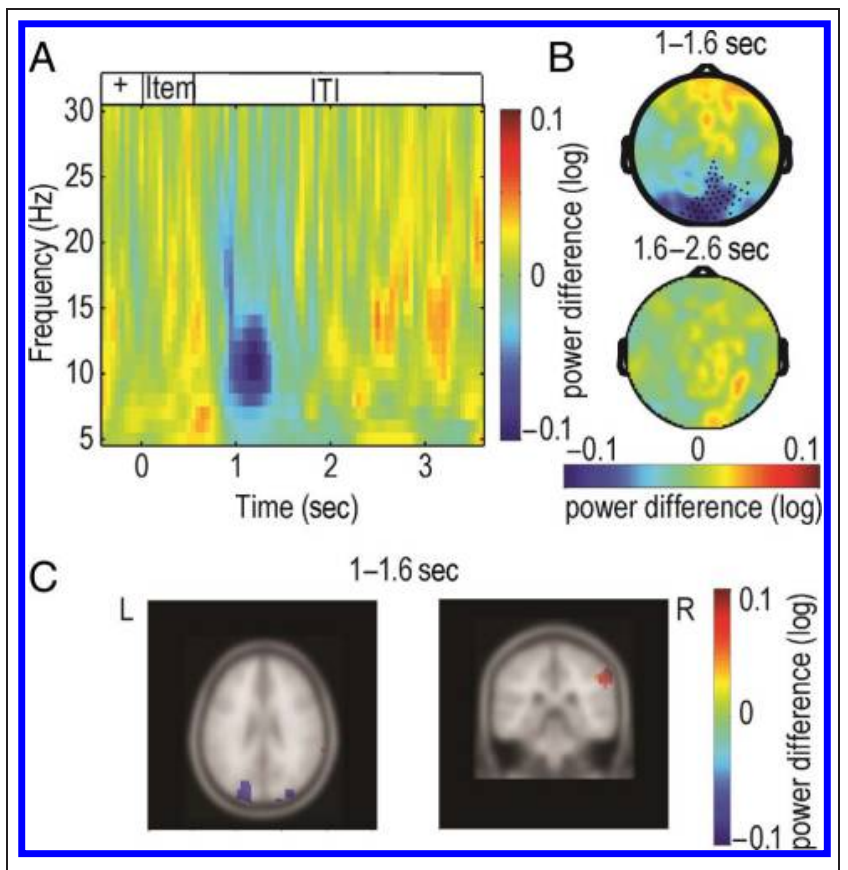

Figure 4. Alpha band activity for Attend-Visual HIT versus AttendVisual MISS. (A) The TFR of power for the contrast Attend-Visual HIT versus Attend-Visual MISS. (B) Scalp topographies of the differences in the 8-12 Hz band for 1-1.6 sec and 1.6-2.6 sec after stimulus onset. Sensors marked with dots are part of the significant clusters obtained in a permutation analysis controlling for multiple comparisons $(p<.05)$. (C) Source reconstruction of the difference in alpha power modulation during the early rehearsal period $(1-1.6 \mathrm{sec})$ in visual cortex and SMG (Attend-Visual HIT vs. Attend-Visual MISS). The regions of significant difference were identified using a cluster-level permutation test $(p<.05)$. This allowed us to control for multiple comparisons in visual cortex and bilateral SMG, respectively. Negative significant clusters in visual cortex and a positive significant cluster in right SMG were found.

sensor level. Summarizing, these results suggest that decreased visual alpha accompanied by increased auditory alpha promotes successful encoding of visually attended items.

\section{Subsequent Memory Effect for Auditory Attended Items}

We then performed the SME analysis for the AttendAuditory condition. Figure 5A and 5B top panels show the TFR of power for the Attend-Auditory HIT versus MISS conditions in the early and late rehearsal, respectively. In the early rehearsal interval, we found a relative alpha decrease over left temporal areas associated with better memory encoding. In the late rehearsal interval, we found increased alpha activity over posterior sensors. A clusterbased randomization test revealed two clusters: a negative cluster over left temporal sensors in the early rehearsal period and a positive cluster over parieto-occipital sensors in the late rehearsal period (1-1.6 sec: $p=.041$; 1.6-2.6 sec: $p=.003$; Figure 5B). The source reflecting memory-related alpha power decrease in the early time 
window was strongest in left SMG ( $p=.044$; Figure 5A, bottom). This might reflect that engagement of the left extended auditory system boosts memory encoding of auditory items. In the late time window, we found sources reflecting memory encoding in the visual cortex $(p=.033$; Figure $5 \mathrm{~B}$, bottom). This is likely to reflect the disengagement of the visual system to prevent distraction during rehearsal. The posterior alpha difference occurs somewhat late after the stimulus offset; however, it is consistent with the findings from Meeuwissen's study (Meeuwissen, Takashima, Fernandez, \& Jensen, 2011).

\section{Incidental Encoding of Visual Items}

We then set out to investigate the neuronal activity associated with the incidental encoding of unattended items. We did this by comparing the TFRs of power for UnattendedVisual HIT versus Unattended-Visual MISS (Figure 6). We observed a highly robust decrease in alpha power over posterior sensors in the memory rehearsal interval (1$2.6 \mathrm{sec}$; Figure 6A). The topography revealed the strongest effect in the alpha band in sensors over occipital regions (1-1.6 sec: $p=.018 ; 1.6-2.6 \mathrm{sec}: p=.01$; Figure 6B). The significant sources of the alpha decrease were found in the visual cortex in the late interval (1.6-2.6 sec; $p=$ .002 ; Figure $6 \mathrm{C}$ ). We propose that the incidental encoding of Unattended-Visual items might be reflected by a relative reduction of occipital alpha band activity reflecting the engagement of the visual system.

\section{Incidental Encoding of Auditory Items}

Next, we investigated the modulations in oscillatory activity associated with the incidental encoding of unattended auditorily presented words. The TFRs of power for the Unattended-Auditory HIT versus Unattended-Auditory MISS contrasts shown in Figure 7A revealed a significant power decrease in the alpha band during the late rehearsal period. The corresponding topographical plots are shown in Figure 7B. When statistically comparing HIT versus MISS for the Unattended-Auditory condition, we found a cluster over occipital-parietal-temporal sensors for the second time window (1.6-2.6 sec; $p=.011)$. Contrary to our expectation, source reconstruction indicated that the power decrease for late rehearsal was produced in the visual areas $(p=.007$; Figure $7 \mathrm{C})$. These could be explained by the incidental encoding of unattended words being reflected by visual imagery in the visual cortex.
Figure 5. Alpha band activity for Attend-Auditory HIT versus Attend-Auditory MISS (A) 1-1.6 sec early rehearsal period. Top: difference in TFRs of power for the contrasts Attend-Auditory HIT versus Attend-Auditory MISS illustrating grand-averaged activity for all participants across sensors of significant clusters (marked by dots in topographical plots). Middle: scalp topographies of the difference in the 8-12 Hz band. Sensors marked with dots are part of significant clusters obtained from a permutation analysis $(p<.05)$. Bottom: source reconstruction of the difference in alpha power in visual cortex and SMG (Attend-Auditory HIT vs. Attend-Auditory MISS).

The regions of significant difference were identified using a cluster-level permutation test $(p<.05)$. This allowed us to control for multiple comparisons in visual cortex and bilateral SMG, respectively. A negative significant cluster in left SMG was found. (B) The 1.6-2.6 sec late rehearsal period. The annotations are similar as described for A.

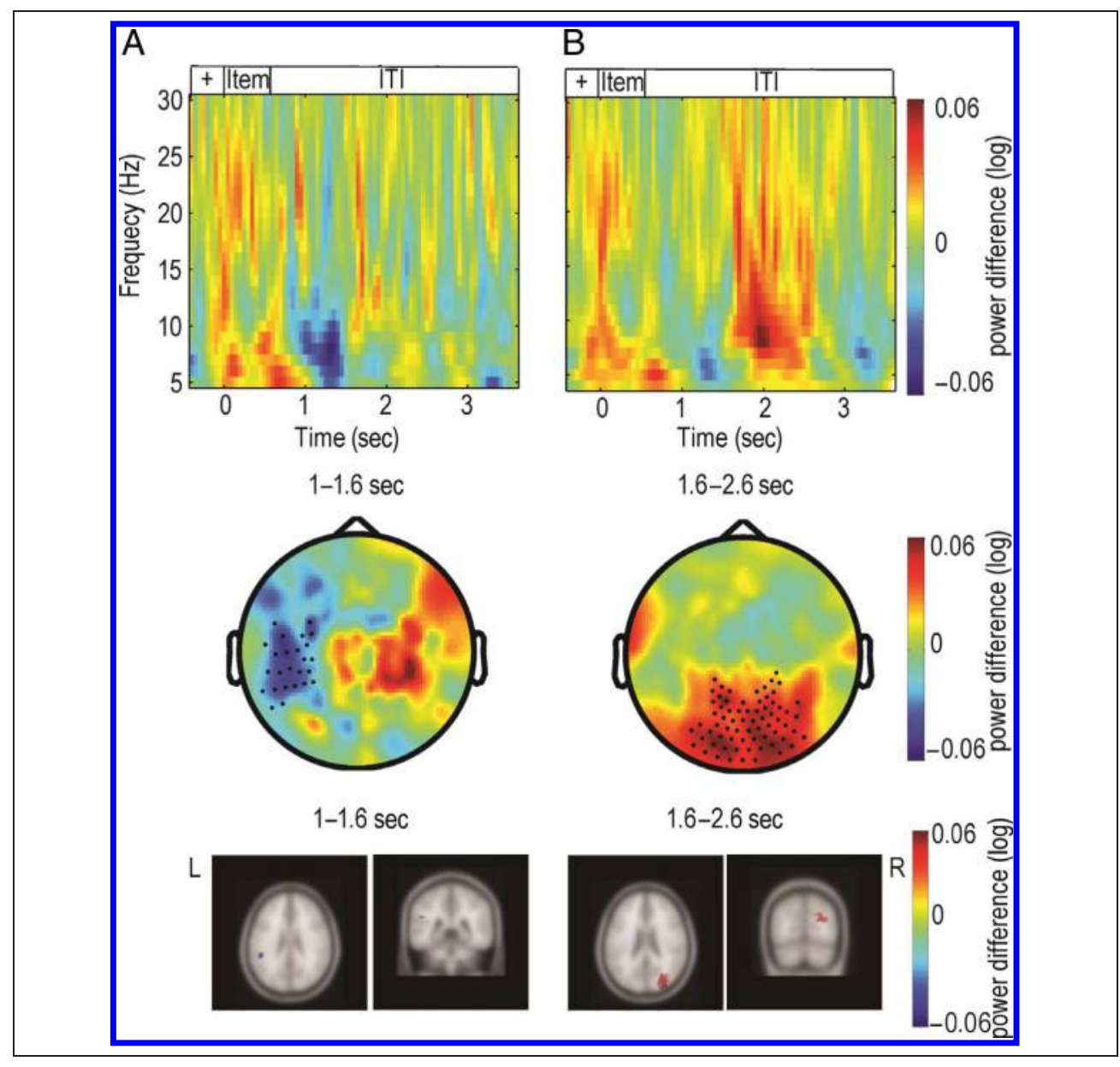




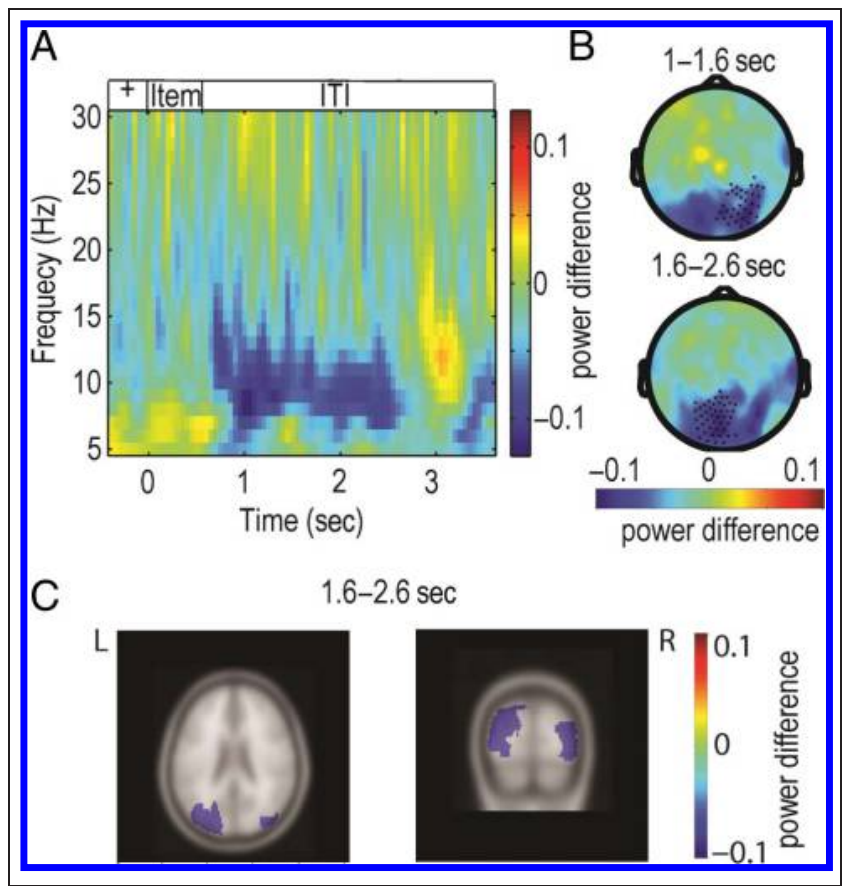

Figure 6. Alpha band activity reflecting the incidental encoding of unattended-visual items, that is, Unattended-Visual HIT versus Unattended-Visual MISS. (A) The TFR of power for the contrast Unattended-Visual HIT versus Unattended-Visual MISS. (B) Scalp topographies of the differences in the 8-12 Hz band for 1-1.6 sec and 1.6-2.6 sec after stimulus onset. Sensors marked with dots are part of the significant clusters obtained in a permutation analysis controlling for multiple comparisons $(p<.05)$. (C) Source reconstruction of the difference in alpha power modulation during the early rehearsal period $(1.6-2.6 \mathrm{sec})$ in visual cortex and SMG (Unattended-Visual HIT vs. Unattended-Visual MISS). The statically reliable sources were identified using a cluster-level permutation test $(p<.05)$. Significant clusters reflecting a negative difference were identified in the visual cortex.

\section{Alpha Power Predicts Differences in Individual Memory Performance}

Lastly, we asked if the modulation in alpha band activity reflected individual memory performance. Across participants, we correlated the difference in posterior alpha power for the Attend-Visual versus Attend-Auditory condition with memory performance ( $d^{\prime}$ value) for the AttendVisual condition (Figure 8A). We selected posterior sensors because posterior parietal alpha modulation significantly correlated with memory performance (Park et al., 2014). We observed a significant negative correlation in the early interval. This suggests that participants who can successfully decrease posterior alpha activity during visual encoding also have better memory for the visual modality. On the contrary, a positive correlation was found in the auditory modality, which was significant in the late interval (Figure 8B). This suggests that participants who can increase their alpha activity during the encoding of auditory items have better memory for that modality. In short, these data suggest that a better ability to modulate posterior alpha activity during memory encoding leads to better subsequent memory performance in a modality-specific manner.

\section{DISCUSSION}

We used MEG to examine modulations of alpha activity in a cross-modal LTM task. We presented participants' auditory (words) and visual (pictures) stimuli simultaneously and asked participants to attend to only one of the modalities. We found that successful LTM encoding was reflected by an alpha power decrease in the sensory region of the to-be-attended modality (Figures 4 and 5). The modulations were predictive of individual memory performance. Moreover, the alpha power increase in the sensory region of the to-be-ignored modality might serve to suppress this modality. This interpretation could be further substantiated in a future EEG/fMRI study by considering if the alpha increase is associated with a BOLD decrease. This suggests that oscillatory alpha band activity reflects the engagement and disengagement of sensory regions in a cross-modal setting supporting LTM encoding.

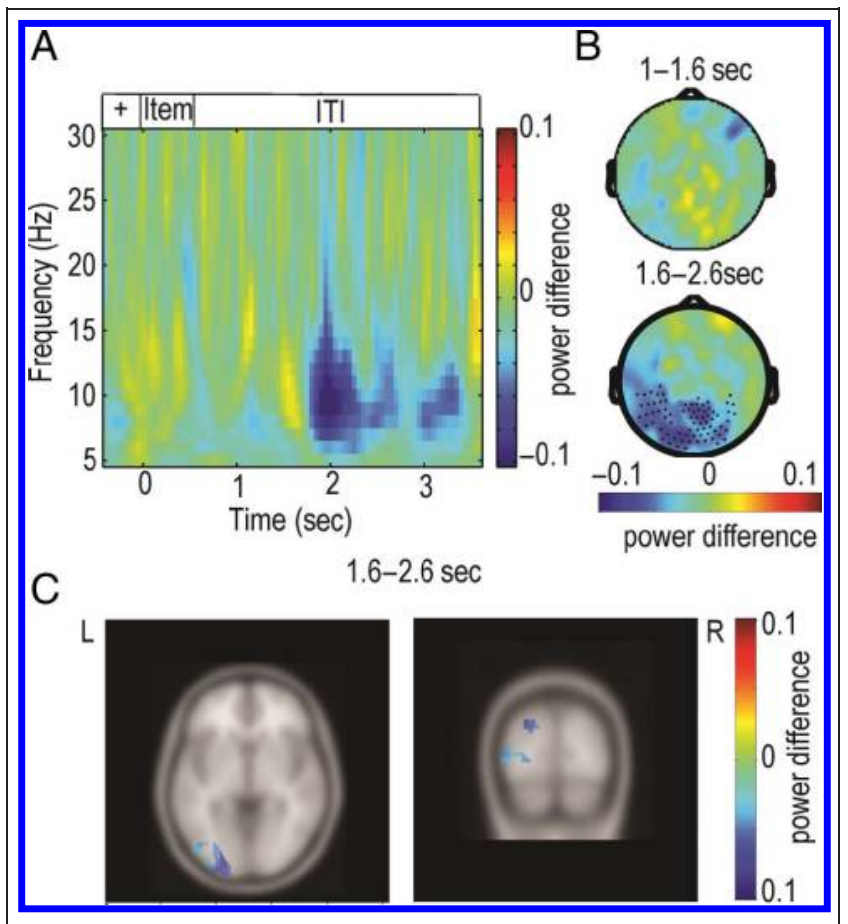

Figure 7. Alpha band activity reflecting the incidental encoding of unattended auditory items, that is, Unattended-auditory HIT versus Unattended-Auditory MISS. (A) The TFR of power for the contrast Unattended-auditory HIT versus Unattended-Auditory MISS. (B) Scalp topographies of the differences in the 8-12 Hz band for 1-1.6 sec and 1.6-2.6 sec after stimulus onset. Sensors marked with dots are part of the significant clusters obtained in a permutation analysis controlling for multiple comparisons $(p<.05)$. (C) Source reconstruction of the difference in alpha power modulation during the early rehearsal period (1.6-2.6 sec; Unattended-Auditory HIT vs. Unattended-Auditory MISS). The statically reliable sources were identified using a cluster-level permutation test $(p<.05)$. Significant clusters reflecting a negative difference were identified in left visual cortex. 


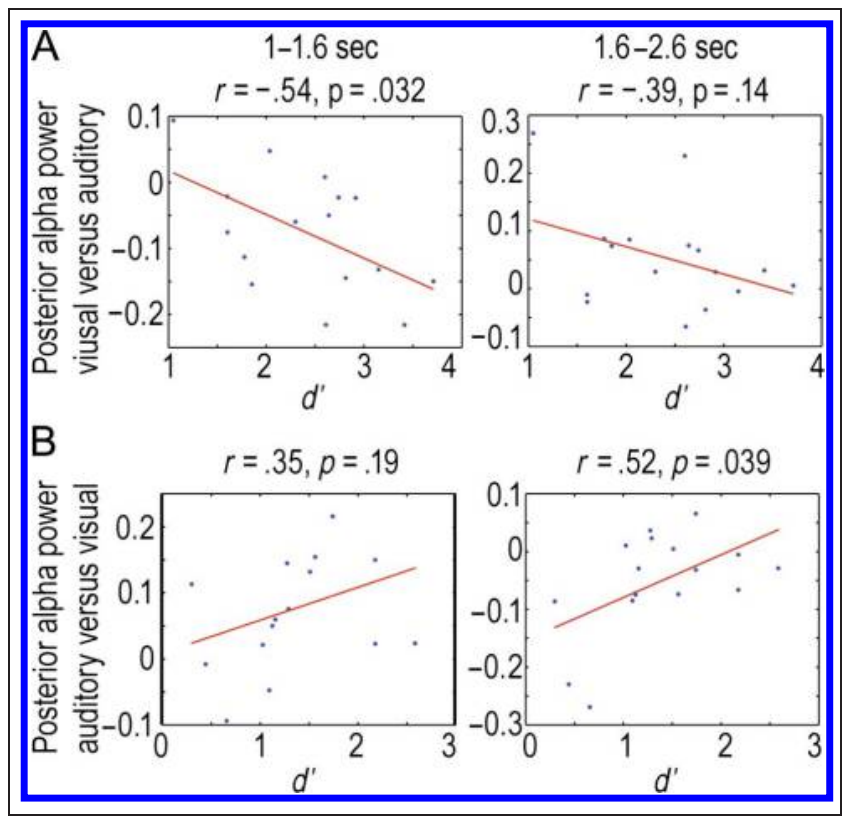

Figure 8. Correlation between alpha band difference between Attend-Visual and Attend-Auditory conditions over posterior sensors during rehearsal and memory performance. (A) Correlation for visual memory performance in early (1-1.6 sec) and late (1.6-2.6 sec) rehearsal periods. (B) Correlation for auditory memory performance in early (1-1.6 sec) and late (1.6-2.6 sec) rehearsal periods.

The notion that alpha oscillatory activity serves to allocate resources during memory encoding is derived from studies on attention (Mazaheri et al., 2014; Thut et al., 2006; Worden et al., 2000), working memory (Dube et al., 2013; Jokisch \& Jensen, 2007; Thut et al., 2006; Sauseng et al., 2005; Klimesch, Doppelmayr, Schwaiger, Auinger, \& Winkler, 1999), and LTM (Park et al., 2014; Waldhauser, Johansson, \& Hanslmayr, 2012; Meeuwissen et al., 2011). On the basis of these findings, we focused specifically on alpha activity in a cross-modal encoding task. The distinct dynamics of alpha power modulation extends the notion that alpha band activity is involved in LTM encoding by modulating the functional engagement and disengagement of sensory specific regions. That is, alpha power decreases in task-relevant areas and increases in task-irrelevant areas (Jensen \& Mazaheri, 2010).

Modulations of alpha band activity with attention and memory has been extensively reported in visual and somatosensory regions. However, recently, alpha band modulations have also been reported in the extended auditory system including the SMG (Mazaheri et al., 2014; Weisz, Muller, Jatzev, \& Bertrand, 2014). Further intracranial recordings have confirmed the existence of alpha activity in the auditory system (Gomez-Ramirez et al., 2011). In our study, we found alpha band modulation in SMG rather than in primary auditory cortex per se. Previous neuroimaging studies have implicated the SMG in higher-level auditoryverbal processing. For instance, left SMG is activated both during speech perception and pitch memory (Sabri et al., 2008; Gaab et al., 2003; Celsis et al., 1999). Furthermore, performance is impaired in a phonological decision task after perturbating the SMG with TMS (Hartwigsen et al., 2010). This has led to the speculation that the left SMG plays a vital role in successful auditory-verbal memory encoding. Thus, we speculate that the increase of alpha in the SMG might reflect the disengagement of the extended auditory-verbal system, which then reflects reduced memory formation for aurally presented items. Conversely, a lack of alpha increase in SMG is taken to facilitate successful encoding of auditory items. This is further confirmed by decreased alpha activity in left SMG in the Attend-Auditory SME. It is, at this stage, not clear to us why we find an alpha decrease reflecting subsequent memory for words in left SMG, whereas when comparing the visual to auditory encoding, we found the right SMG to be implicated. Possibly this is explained by left hemisphere dominance in language-related function processing (Ojemann, Ojemann, Lettich, \& Berger, 1989). It should also be mentioned that the lateral posterior parietal cortex (PPC), which includes the SMG, has been implicated in LTM processes in several brain imaging studies (Uncapher \& Wagner, 2009). From these studies, the view has emerged that increased activation in dorsal PPC is associated with later memory success whereas the engagement of ventral lateral PPC reflects later memory failure. Assuming that increased alpha activity reflects disengagement, this notion is not easily reconciled with our findings pertaining to subsequent memory in the left SMG. However, the difference in alpha power in the right SMG when comparing the visual to auditory contrast might reflect a differential engagement of the ventral lateral PPC associated with supramodal memory encoding (Uncapher \& Wagner, 2009).

Several M/EEG studies have shown that decreased alpha activity (Park et al., 2014; Hanslmayr, Spitzer, \& Bauml, 2009; Spitzer, Hanslmayr, Opitz, Mecklinger, \& Bauml, 2009; Sederberg et al., 2006) as well as increased alpha activity (Meeuwissen et al., 2011; Khader, Jost, Ranganath, \& Rosler, 2010) are related to successful memory encoding. This seems to paint a rather complex picture of the relation between brain oscillations and memory formation. In Meeuwissen's study (Meeuwissen et al., 2011), participants were instructed to retrieve the order of three words, and they found that parieto-occipital alpha power during the rehearsal period was markedly stronger for successfully than unsuccessfully encoded LTM sequences. The rehearsal of the words probably relied heavily on language-related areas and thus was associated with a strong posterior alpha increase. A study by Park et al. (2014) used a paradigm in which a cue indicated where an upcoming picture should be encoded or ignored. In line with our findings, they demonstrated that alpha power in posterior regions served to gate encoding. Besides, incidental encoding was associated with an insufficient alpha increase. Importantly, they quantified the cuing interval before the onset of the memory items. In our study, we applied a cross-modal paradigm in which we characterized the oscillatory brain activity after item onset, that is, the 
encoding and rehearsal period. Thus, we found that the anticipatory effects identified by Park et al. extend into the encoding and rehearsal period for both the visual and auditory modality.

Attention and memory are likely to share overlapping mechanisms (Awh \& Jonides, 2001; Norman, 1968). This is supported by the correlation between posterior alpha difference during the visual versus auditory attention contrast and memory performance, that is, a negative correlation in visual memory performance and a positive correlation in auditory memory performance. This suggests that modality-specific changes in alpha activity reflect attentional modulation, which facilitates successful LTM encoding. These results also suggest that difficulties in prioritizing attention and suppressing irrelevant information in elderly people (Guerreiro, Murphy, \& Van Gerven, 2010) might be because of an inability to successfully modulate alpha activity.

Our findings provide evidence that alpha activity gates LTM encoding. Successful memory encoding is facilitated if alpha activity decreases in task-relevant areas to encode relevant information and increases in task-irrelevant areas to block out irrelevant information. To further explore the mechanistic underpinnings of these findings, future work may involve TMS to entrain visual regions at $\sim 10 \mathrm{~Hz}$ in the auditory encoding condition to investigate the possible enhancement of auditory memory performance. This will allow us to determine a causal role of the alpha band activity for attentional allocation, supporting memory encoding.

In conclusion, our study adds to a growing body of evidence demonstrating that gating to different regions is reflected by local alpha band activity. We have here demonstrated that this mechanism also works in a modalityspecific manner when LTM representation must be encoded and rehearsed.

\section{Acknowledgments}

We would like to thank Marieke van de Nieuwenhuijzen for her invaluable comments on an earlier version of the manuscript. Haiteng Jiang has received support from the China Scholarship Council. Ole Jensen is funded by a VICI grant (453-09-002) and 'The healthy brain' funded by the Netherlands Initiative Brain and Cognitive (NIHC), a part of the Organization for Scientific Research (NWO) (056-14-011).

Reprint requests should be sent to Haiteng Jiang, Donders Institute for Brain, Cognition and Behaviour, Radboud University Nijmegen, Kapittelweg 29, Nijmegen, Netherlands, 6525 EN, or via e-mail: h.jiang@fcdonders.ru.nl.

\section{REFERENCES}

Adrian, E. D. (1944). Brain rhythms. Nature, 153, 360-362. Awh, E., \& Jonides, J. (2001). Overlapping mechanisms of attention and spatial working memory. Trends in Cognitive Sciences, 5, 119-126.

Celsis, P., Boulanouar, K., Doyon, B., Ranjeva, J. P., Berry, I., Nespoulous, J. L., et al. (1999). Differential fMRI responses in the left posterior superior temporal gyrus and left supramarginal gyrus to habituation and change detection in syllables and tones. Neuroimage, 9, 135-144.

Dube, C., Payne, L., Sekuler, R., \& Rotello, C. M. (2013). Paying attention to attention in recognition memory: Insights from models and electrophysiology. Psychological Science, 24, 2398-2408.

Foxe, J. J., Simpson, G. V., \& Ahlfors, S. P. (1998). Parietooccipital similar to $10 \mathrm{~Hz}$ activity reflects anticipatory state of visual attention mechanisms. NeuroReport. 9, 3929-3933.

Foxe, J. J., \& Snyder, A. C. (2011). The role of alpha-band brain oscillations as a sensory suppression mechanism during selective attention. Frontiers in Psychology, 2.

Fu, K. M. G., Foxe, J. J., Murray, M. M., Higgins, B. A., Javitt, D. C., \& Schroeder, C. E. (2001). Attention-dependent suppression of distracter visual input can be cross-modally cued as indexed by anticipatory parieto-occipital alpha-band oscillations. Cognitive Brain Research, 12, 145-152.

Gaab, N., Gaser, C., Zaehle, T., Jancke, L., \& Schlaug, G. (2003). Functional anatomy of pitch memory-An fMRI study with sparse temporal sampling. Neuroimage, 19, 1417-1426.

Gomez-Ramirez, M., Kelly, S. P., Molholm, S., Sehatpour, P., Schwartz, T. H., \& Foxe, J. J. (2011). Oscillatory sensory selection mechanisms during intersensory attention to rhythmic auditory and visual inputs: A human electrocorticographic investigation. Journal of Neuroscience, 31, 18556-18567.

Gross, J., Kujala, J., Hamalainen, M., Timmermann, L., Schnitzler, A., \& Salmelin, R. (2001). Dynamic imaging of coherent sources: Studying neural interactions in the human brain. Proceedings of the National Academv of Sciences. U.S.A., 98, 694-699.

Guerreiro, M. J. S., Murphy, D. R., \& Van Gerven, P. W. M. (2010). The role of sensory modality in age-related distraction: A critical review and a renewed view. Psychological Bulletin, 136, 975-1022.

Haegens, S., Handel, B. F., \& Jensen, O. (2011). Top-down controlled alpha band activity in somatosensory areas determines behavioral performance in a discrimination task. Journal of Neuroscience, 31, 5197-5204.

Haegens, S., Luther, L., \& Jensen, O. (2012). Somatosensory anticipatory alpha activity increases to suppress distracting input. Iournal of Cognitive Neuroscience, 24, 677-685.

Handel, B. F., Haarmeier, T., \& Jensen, O. (2011). Alpha oscillations correlate with the successful inhibition of unattended stimuli. Lournal of Cognitive Neuroscience. 23, 2494-2502.

Hanslmayr, S., Spitzer, B., \& Bauml, K. H. (2009). Brain oscillations dissociate between semantic and nonsemantic encoding of episodic memories. Cerebral Cortex, 19, 1631-1640.

Hartwigsen, G., Baumgaertner, A., Price, C. J., Koehnke, M., Ulmer, S., \& Siebner, H. R. (2010). Phonological decisions require both the left and right supramarginal gyri. Proceedings of the National Academv of Sciences. U.S.A., 107, 16494-16499.

Jensen, O., \& Mazaheri, A. (2010). Shaping functional architecture by oscillatory alpha activity: Gating by inhibition. Frontiers in Human Neuroscience, 4.

Jokisch, D., \& Jensen, O. (2007). Modulation of gamma and alpha activity during a working memory task engaging the dorsal or ventral stream. Iournal of Neuroscience, 27, 3244-3251.

Kelly, S. P., Lalor, E. C., Reilly, R. B., \& Foxe, J. J. (2006). Increases in alpha oscillatory power reflect an active retinotopic mechanism for distracter suppression during 
sustained visuospatial attention. Iournal of Neuropbysiology. 95, 3844-3851.

Khader, P. H., Jost, K., Ranganath, C., \& Rosler, F. (2010). Theta and alpha oscillations during working-memory maintenance predict successful long-term memory encoding. Neuroscience Letters, 468, 339-343.

Klimesch, W. (2012). Alpha-band oscillations, attention, and controlled access to stored information. Trends in Cognitive Sciences, 16, 606-617.

Klimesch, W., Doppelmayr, M., Schwaiger, J., Auinger, P., \& Winkler, T. (1999). "Paradoxical" alpha synchronization in a memory task. Cognitive Brain Research, 7, 493-501.

Klimesch, W., Sauseng, P., \& Hanslmayr, S. (2007). EEG alpha oscillations: The inhibition-timing hypothesis. Brain Research Reviews, 53, 63-88.

Maris, E., \& Oostenveld, R. (2007). Nonparametric statistical testing of EEG- and MEG-data. Journal of Neuroscience Methods, 164, 177-190.

Mazaheri, A., van Schouwenburg, M. R., Dimitrijevic, A., Denys, D., Cools, R., \& Jensen, O. (2014). Region-specific modulations in oscillatory alpha activity serve to facilitate processing in the visual and auditory modalities.

Neuroimage, 87, 356-362.

Medendorp, W. P., Kramer, G. F. I., Jensen, O., Oosterveld, R., Schoffelen, J. M., \& Fries, P. (2007). Oscillatory activity in human parietal and occipital cortex shows hemispheric lateralization and memory effects in a delayed double-step saccade task. Cerebral Cortex, 17, 2364-2374.

Meeuwissen, E. B., Takashima, A., Fernandez, G., \& Jensen, O. (2011). Increase in posterior alpha activity during rehearsal predicts successful long-term memory formation of word sequences. Human Brain Mapping, 32, 2045-2053.

Norman, D. A. (1968). Toward a theory of memory and attention. Psvchological Review, 75, 522-536.

Ojemann, G., Ojemann, J., Lettich, E., \& Berger, M. (1989). Cortical language localization in left, dominant hemisphere-An electrical-stimulation mapping investigation in 117 patients. Journal of Neurosurgery $71,316-326$

Oostenveld, R., Fries, P., Maris, E., \& Schoffelen, J. M. (2011) FieldTrip: Open source software for advanced analysis of MEG, EEG, and invasive electrophysiological data. Computational Intelligence and Neuroscience, 2011, 156869.

Park, H., Lee, D. S., Kang, E., Kang, H., Hahm, J., Kim, J. S., et al. (2014). Blocking of irrelevant memories by posterior alpha activity boosts memory encoding. Human Brain Madping 35, 3972-3987.

Payne, L., Guillory, S., \& Sekuler, R. (2013). Attention-modulated alpha-band oscillations protect against intrusion of irrelevant information. Iournal of Cognitive Neuroscience, 25 , 1463-1476.

Sabri, M., Binder, J. R., Desai, R., Medler, D. A., Leitl, M. D., \& Liebenthal, E. (2008). Attentional and linguistic interactions in speech perception. Neuroimage 39, 1444-1456.

Sauseng, P., Klimesch, W., Doppelmayr, M., Pecherstorfer, T., Freunberger, R., \& Hanslmayr, S. (2005). EEG alpha synchronization and functional coupling during top-down processing in a working memory task. Human Brain Mapping, 26, 148-155.

Sederberg, P. B., Gauthier, L. V., Terushkin, V., Miller, J. F., Barnathan, J. A., \& Kahana, M. J. (2006). Oscillatory correlates of the primacy effect in episodic memory. Neuroimage 32, 1422-1431.

Spitzer, B., Hanslmayr, S., Opitz, B., Mecklinger, A., \& Bauml, K. H. (2009). Oscillatory correlates of retrieval-induced forgetting in recognition memory. Journal of Cognitive Neuroscience, 21, 976-990.

Stolk, A., Todorovic, A., Schoffelen, J. M., \& Oostenveld, R. (2013). Online and offline tools for head movement compensation in MEG. Neuroimage, 68, 39-48.

Thut, G., Nietzel, A., Brandt, S. A., \& Pascual-Leone, A. (2006). Alpha-band electroencephalographic activity over occipital cortex indexes visuospatial attention bias and predicts visual target detection. Journal of Neuroscience, 26, 9494-9502.

Tzourio-Mazoyer, N., Landeau, B., Papathanassiou, D., Crivello, F., Etard, O., Delcroix, N., et al. (2002). Automated anatomical labeling of activations in SPM using a macroscopic anatomical parcellation of the MNI MRI single-subject brain. Neuroimage, 15, 273-289.

Uncapher, M. R., \& Wagner, A. D. (2009). Posterior parietal cortex and episodic encoding: Insights from fMRI subsequent memory effects and dual-attention theory. Neurobiology of Learning and Memorv, 91, 139-154.

Waldhauser, G. T., Johansson, M., \& Hanslmayr, S. (2012). Alpha/beta oscillations indicate inhibition of interfering visual memories. Iournal of Neuroscience, 32, 1953-1961.

Weisz, N., Muller, N., Jatzev, S., \& Bertrand, O. (2014). Oscillatory alpha modulations in right auditory regions reflect the validity of acoustic cues in an auditory spatial attention task. Cerebral Cortex, 24, 2579-2590.

Willenbockel, V., Sadr, J., Fiset, D., Horne, G. O., Gosselin, F., \& Tanaka, J. W. (2010). Controlling low-level image properties: The SHINE toolbox. Behavior Research Methods, 42, 671-684.

Worden, M. S., Foxe, J. J., Wang, N., \& Simpson, G. V. (2000). Anticipatory biasing of visuospatial attention indexed by retinotopically specific alpha-band electroencephalography increases over occipital cortex. Journal of Neuroscience. 20. RC63. 


\section{This article has been cited by:}

1. Thomas Kustermann, Brigitte Rockstroh, Gregory A. Miller, Tzvetan Popov. 2018. Neural network communication facilitates verbal working memory. Biological Psychology 136, 119-126. [Crossref]

2. M. Beardsley, D. Hernández-Leo, R. Ramirez-Melendez. 2018. Seeking reproducibility: Assessing a multimodal study of the testing effect. Journal of Computer Assisted Learning 22. . [Crossref]

3. Tamas Minarik, Barbara Berger, Paul Sauseng. 2017. The involvement of alpha oscillations in voluntary attention directed towards encoding episodic memories. NeuroImage . [Crossref]

4. Theofilos Petsas, Jemma Harrison, Makio Kashino, Shigeto Furukawa, Maria Chait. 2016. The effect of distraction on change detection in crowded acoustic scenes. Hearing Research 341, 179-189. [Crossref]

5. Tony W. Wilson, Elizabeth Heinrichs-Graham, Amy L. Proskovec, Timothy J. McDermott. 2016. Neuroimaging with magnetoencephalography: A dynamic view of brain pathophysiology. Translational Research 175, 17-36. [Crossref]

6. Amy L. Proskovec, Elizabeth Heinrichs-Graham, Tony W. Wilson. 2016. Aging modulates the oscillatory dynamics underlying successful working memory encoding and maintenance. Human Brain Mapping 37:6, 2348-2361. [Crossref]

7. H. Jiang, T. Popov, P. Jylänki, K. Bi, Z. Yao, Q. Lu, O. Jensen, M.A.J. van Gerven. 2016. Predictability of depression severity based on posterior alpha oscillations. Clinical Neurophysiology 127:4, 2108-2114. [Crossref]

8. Elizabeth Heinrichs-Graham, Tony W. Wilson. 2015. Spatiotemporal oscillatory dynamics during the encoding and maintenance phases of a visual working memory task. Cortex 69, 121-130. [Crossref] 